\title{
Congruences and Quotient Algebras of BCI-algebras
}

\author{
Yuzhong Ding \\ Qingdao University of Science \\ and Technology \\ China
}

\author{
Zhiyong Pang \\ Qingdao University of Science \\ and Technology \\ China
}

Summary. We have formalized the BCI-algebras closely following the book [7] pp.16-19 and pp.58-65. Firstly, the article focuses on the properties of the element and then the definition and properties of congruences and quotient algebras are given. Quotient algebras are the basic tools for exploring the structures of BCI-algebras.

MML identifier: BCIALG_2, version: 7.8.05 4.87.985

The articles [11], [5], [12], [10], [13], [2], [3], [1], [8], [14], [6], [15], [4], and [9] provide the terminology and notation for this paper.

\section{Basic Properties of the Element}

For simplicity, we adopt the following convention: $X$ is a BCI-algebra, $I$ is an ideal of $X, a, x, y, z, u$ are elements of $X, f$ is a function from $\mathbb{N}$ into the carrier of $X$, and $j, i, k, n, m$ are elements of $\mathbb{N}$.

Let us consider $X, x, y$ and let $n$ be an element of $\mathbb{N}$. The functor $(x \backslash y)^{n}$ yielding an element of $X$ is defined by:

(Def. 1) There exists $f$ such that $(x \backslash y)^{n}=f(n)$ and $f(0)=x$ and for every element $j$ of $\mathbb{N}$ such that $j<n$ holds $f(j+1)=f(j) \backslash y$.

One can prove the following propositions:

(1) $(x \backslash y)^{0}=x$.

(2) $(x \backslash y)^{1}=x \backslash y$.

(3) $(x \backslash y)^{2}=x \backslash y \backslash y$. 
(4) $(x \backslash y)^{n+1}=\left((x \backslash y)^{n}\right) \backslash y$.

(5) $\left(x \backslash 0_{X}\right)^{n+1}=x$.

(6) $\left(0_{X} \backslash 0_{X}\right)^{n}=0_{X}$.

(7) $\left((x \backslash y)^{n}\right) \backslash z=((x \backslash z) \backslash y)^{n}$.

(8) $\quad(x \backslash(x \backslash(x \backslash y)))^{n}=(x \backslash y)^{n}$.

(9) $\quad\left(\left(0_{X} \backslash x\right)^{n}\right)^{\mathrm{c}}=\left(0_{X} \backslash x^{\mathrm{c}}\right)^{n}$.

(10) $\quad\left((x \backslash y)^{n} \backslash y\right)^{m}=(x \backslash y)^{n+m}$.

(11) $\left((x \backslash y)^{n} \backslash z\right)^{m}=\left((x \backslash z)^{m} \backslash y\right)^{n}$.

(12) $\quad\left(\left(\left(0_{X} \backslash x\right)^{n}\right)^{\mathrm{c}}\right)^{\mathrm{c}}=\left(0_{X} \backslash x\right)^{n}$.

(13) $\left(0_{X} \backslash x\right)^{n+m}=\left(\left(0_{X} \backslash x\right)^{n}\right) \backslash\left(\left(0_{X} \backslash x\right)^{m}\right)^{\mathrm{c}}$.

(14) $\quad\left(\left(0_{X} \backslash x\right)^{m+n}\right)^{\mathrm{c}}=\left(\left(0_{X} \backslash x\right)^{m}\right)^{\mathrm{c}} \backslash\left(\left(0_{X} \backslash x\right)^{n}\right)$.

(15) $\quad\left(\left(0_{X} \backslash\left(\left(0_{X} \backslash x\right)^{m}\right)\right)^{n}\right)^{\mathrm{c}}=\left(0_{X} \backslash x\right)^{m \cdot n}$.

(16) If $\left(0_{X} \backslash x\right)^{m}=0_{X}$, then $\left(0_{X} \backslash x\right)^{m \cdot n}=0_{X}$.

(17) If $x \backslash y=x$, then $(x \backslash y)^{n}=x$.

(18) $\quad\left(0_{X} \backslash(x \backslash y)\right)^{n}=\left(\left(0_{X} \backslash x\right)^{n}\right) \backslash\left(\left(0_{X} \backslash y\right)^{n}\right)$.

(19) If $x \leq y$, then $(x \backslash z)^{n} \leq(y \backslash z)^{n}$.

(20) If $x \leq y$, then $(z \backslash y)^{n} \leq(z \backslash x)^{n}$.

(21) $\left((x \backslash z)^{n}\right) \backslash\left((y \backslash z)^{n}\right) \leq x \backslash y$.

(22) $\quad\left((x \backslash(x \backslash y))^{n} \backslash(y \backslash x)\right)^{n} \leq x$.

Let us consider $X, a$. We introduce $a$ is minimal as a synonym of $a$ is atom.

Let us consider $X, a$. We say that $a$ is positive if and only if:

(Def. 2) $0_{X} \leq a$.

We say that $a$ is least if and only if:

(Def. 3) For every $x$ holds $a \leq x$.

We say that $a$ is maximal if and only if:

(Def. 4) For every $x$ such that $a \leq x$ holds $x=a$.

We say that $a$ is greatest if and only if:

(Def. 5) For every $x$ holds $x \leq a$.

Let us consider $X$. Observe that there exists an element of $X$ which is positive.

Let us consider $X$. Note that $0_{X}$ is positive and minimal.

Next we state several propositions:

(23) $a$ is minimal iff for every $x$ holds $a \backslash x=x^{\mathrm{c}} \backslash a^{\mathrm{c}}$.

(24) $x^{\mathrm{c}}$ is minimal iff for every $y$ such that $y \leq x$ holds $x^{\mathrm{c}}=y^{\mathrm{c}}$.

(25) $x^{\mathrm{c}}$ is minimal iff for all $y, z$ holds $\left((x \backslash z \backslash(y \backslash z))^{\mathrm{c}}\right)^{\mathrm{c}}=y^{\mathrm{c}} \backslash x^{\mathrm{c}}$.

(26) If $0_{X}$ is maximal, then every $a$ is minimal.

(27) If there exists $x$ which is greatest, then every $a$ is positive. 
(28) $x \backslash\left(x^{\mathrm{c}}\right)^{\mathrm{c}}$ is a positive element of $X$.

(29) $a$ is minimal iff $\left(a^{\mathrm{c}}\right)^{\mathrm{c}}=a$.

(30) $a$ is minimal iff there exists $x$ such that $a=x^{\mathrm{c}}$.

Let us consider $X, x$. We say that $x$ is nilpotent if and only if:

(Def. 6) There exists a non empty element $k$ of $\mathbb{N}$ such that $\left(0_{X} \backslash x\right)^{k}=0_{X}$.

Let us consider $X$. We say that $X$ is nilpotent if and only if:

(Def. 7) Every element of $X$ is nilpotent.

Let us consider $X, x$. Let us assume that $x$ is nilpotent. The functor ord $(x)$ yielding a non empty element of $\mathbb{N}$ is defined by:

(Def. 8) $\quad\left(0_{X} \backslash x\right)^{\operatorname{ord}(x)}=0_{X}$ and for every element $m$ of $\mathbb{N}$ such that $\left(0_{X} \backslash x\right)^{m}=0_{X}$ and $m \neq 0$ holds ord $(x) \leq m$.

Let us consider $X$. One can verify that $0_{X}$ is nilpotent.

We now state four propositions:

(31) $x$ is a positive element of $X$ iff $x$ is nilpotent and $\operatorname{ord}(x)=1$.

(32) $X$ is a BCK-algebra iff for every $x$ holds ord $(x)=1$ and $x$ is nilpotent.

(33) $\left(0_{X} \backslash x^{\mathrm{c}}\right)^{n}$ is minimal.

(34) If $x$ is nilpotent, then $\operatorname{ord}(x)=\operatorname{ord}\left(x^{\mathrm{c}}\right)$.

\section{Congruences and Quotient Algebras}

Let $X$ be a BCI-algebra. An equivalence relation of $X$ is said to be a congruence of $X$ if:

(Def. 9) For all elements $x, y, u, v$ of $X$ such that $\langle x, y\rangle \in$ it and $\langle u, v\rangle \in$ it holds $\langle x \backslash u, y \backslash v\rangle \in$ it.

Let $X$ be a BCI-algebra. An equivalence relation of $X$ is said to be an L-congruence of $X$ if:

(Def. 10) For all elements $x, y$ of $X$ such that $\langle x, y\rangle \in$ it and for every element $u$ of $X$ holds $\langle u \backslash x, u \backslash y\rangle \in$ it.

Let $X$ be a BCI-algebra. An equivalence relation of $X$ is said to be an R-congruence of $X$ if:

(Def. 11) For all elements $x, y$ of $X$ such that $\langle x, y\rangle \in$ it and for every element $u$ of $X$ holds $\langle x \backslash u, y \backslash u\rangle \in$ it.

Let $X$ be a BCI-algebra and let $A$ be an ideal of $X$. A binary relation on $X$ is said to be an I-congruence of $X$ by $A$ if:

(Def. 12) For all elements $x, y$ of $X$ holds $\langle x, y\rangle \in$ it iff $x \backslash y \in A$ and $y \backslash x \in A$.

Let $X$ be a BCI-algebra and let $A$ be an ideal of $X$. Note that every Icongruence of $X$ by $A$ is total, symmetric, and transitive.

Let $X$ be a BCI-algebra. The functor IConSet $X$ is defined as follows: 
(Def. 13) For every set $A_{1}$ holds $A_{1} \in$ IConSet $X$ iff there exists an ideal $I$ of $X$ such that $A_{1}$ is an I-congruence of $X$ by $I$.

Let $X$ be a BCI-algebra. The functor ConSet $X$ is defined as follows:

(Def. 14) ConSet $X=\{R: R$ ranges over congruences of $X\}$.

The functor LConSet $X$ is defined by:

(Def. 15) LConSet $X=\{R: R$ ranges over L-congruences of $X\}$.

The functor RConSet $X$ is defined as follows:

(Def. 16) RConSet $X=\{R: R$ ranges over R-congruences of $X\}$.

For simplicity, we adopt the following rules: $R$ is an equivalence relation of $X, R_{1}$ is an I-congruence of $X$ by $I, E$ is a congruence of $X, R_{2}$ is an R-congruence of $X$, and $L_{1}$ is an L-congruence of $X$.

We now state three propositions:

(35) For all $X, E$ holds $\left[0_{X}\right]_{E}$ is a closed ideal of $X$.

(36) $R$ is a congruence of $X$ iff $R$ is an R-congruence of $X$ and an L-congruence of $X$.

(37) $\quad R_{1}$ is a congruence of $X$.

Let $X$ be a BCI-algebra and let $I$ be an ideal of $X$. We see that the Icongruence of $X$ by $I$ is a congruence of $X$.

One can prove the following propositions:

(38) $\left[0_{X}\right]_{\left(R_{1}\right)} \subseteq I$.

(39) $I$ is closed iff $I=\left[0_{X}\right]_{\left(R_{1}\right)}$.

(40) If $\langle x, y\rangle \in E$, then $x \backslash y \in\left[0_{X}\right]_{E}$ and $y \backslash x \in\left[0_{X}\right]_{E}$.

(41) Let $A, I$ be ideals of $X, I_{1}$ be an I-congruence of $X$ by $A$, and $I_{2}$ be an I-congruence of $X$ by $I$. If $\left[0_{X}\right]_{\left(I_{1}\right)}=\left[0_{X}\right]_{\left(I_{2}\right)}$, then $I_{1}=I_{2}$.

(42) If $\langle x, y\rangle \in E$ and $u \in\left[0_{X}\right]_{E}$, then $\left\langle x,(y \backslash u)^{k}\right\rangle \in E$.

(43) Suppose that for all $X, x, y$ there exist $i, j, m, n$ such that $((x \backslash(x \backslash$ $\left.y))^{i} \backslash(y \backslash x)\right)^{j}=\left((y \backslash(y \backslash x))^{m} \backslash(x \backslash y)\right)^{n}$. Let given $E$, I. If $I=\left[0_{X}\right]_{E}$, then $E$ is an I-congruence of $X$ by $I$.

(44) IConSet $X \subseteq$ ConSet $X$.

(45) ConSet $X \subseteq$ LConSet $X$.

(46) ConSet $X \subseteq$ RConSet $X$.

(47) $\operatorname{ConSet} X=$ LConSet $X \cap \operatorname{RConSet} X$.

(48) If every $L_{1}$ is an I-congruence of $X$ by $I$, then $E=R_{1}$.

(49) If every $R_{2}$ is an I-congruence of $X$ by $I$, then $E=R_{1}$.

(50) $\left[0_{X}\right]_{\left(L_{1}\right)}$ is a closed ideal of $X$.

In the sequel $E$ denotes a congruence of $X$ and $R_{1}$ denotes an I-congruence of $X$ by $I$.

Let us consider $X, E$. Note that Classes $E$ is non empty. 
Let us consider $X, E$. The functor EqClaOp $E$ yielding a binary operation on Classes $E$ is defined by:

(Def. 17) For all elements $W_{1}, W_{2}$ of Classes $E$ and for all $x, y$ such that $W_{1}=[x]_{E}$ and $W_{2}=[y]_{E}$ holds $(\mathrm{EqClaOp} E)\left(W_{1}, W_{2}\right)=[x \backslash y]_{E}$.

Let us consider $X, E$. The functor zeroEqC $E$ yields an element of Classes $E$ and is defined as follows:

(Def. 18) zeroEqC $E=\left[0_{X}\right]_{E}$.

Let us consider $X, E$. The functor $X / E$ yielding a BCI structure with 0 is defined by:

(Def. 19) $X /{ }_{E}=\langle$ Classes $E$, EqClaOp $E$, zeroEqC $E\rangle$.

Let us consider $X$ and let $E$ be a congruence of $X$. One can check that ${ }^{X} / E$ is non empty.

In the sequel $W_{1}, W_{2}$ denote elements of Classes $E$.

Let us consider $X, E, W_{1}, W_{2}$. The functor $W_{1} \backslash W_{2}$ yielding an element of Classes $E$ is defined by:

(Def. 20) $\quad W_{1} \backslash W_{2}=(\mathrm{EqClaOp} E)\left(W_{1}, W_{2}\right)$.

Next we state the proposition

(51) $X / R_{1}$ is a BCI-algebra.

Let us consider $X, I, R_{1}$. Note that $X / R_{1}$ is strict, B, C, I, and BCI-4.

Next we state the proposition

(52) For all $X, I$ such that $I=$ BCK-part $X$ and for every I-congruence $R_{1}$ of $X$ by $I$ holds $X / R_{1}$ is a $p$-semisimple BCI-algebra.

\section{REFERENCES}

[1] Czesław Byliński. Binary operations. Formalized Mathematics, 1(1):175-180, 1990.

[2] Czesław Byliński. Functions and their basic properties. Formalized Mathematics, 1(1):5565, 1990.

[3] Czesław Byliński. Functions from a set to a set. Formalized Mathematics, 1(1):153-164, 1990.

[4] Czesław Byliński. Partial functions. Formalized Mathematics, 1(2):357-367, 1990.

[5] Czesław Byliński. Some basic properties of sets. Formalized Mathematics, 1(1):47-53, 1990.

[6] Yuzhong Ding. Several classes of BCI-algebras and their properties. Formalized Mathematics, 15(1):1-9, 2007.

[7] Yisheng Huang. BCI-algebras. Science Press, 2006.

[8] Library Committee of the Association of Mizar Users. Binary operations on numbers. To appear in Formalized Mathematics.

[9] Konrad Raczkowski and Paweł Sadowski. Equivalence relations and classes of abstraction. Formalized Mathematics, 1(3):441-444, 1990.

[10] Andrzej Trybulec. Subsets of complex numbers. To appear in Formalized Mathematics.

[11] Andrzej Trybulec. Tarski Grothendieck set theory. Formalized Mathematics, 1(1):9-11, 1990

[12] Zinaida Trybulec. Properties of subsets. Formalized Mathematics, 1(1):67-71, 1990.

[13] Edmund Woronowicz. Relations and their basic properties. Formalized Mathematics, 1(1):73-83, 1990.

[14] Edmund Woronowicz. Relations defined on sets. Formalized Mathematics, 1(1):181-186, 1990. 
[15] Edmund Woronowicz and Anna Zalewska. Properties of binary relations. Formalized Mathematics, 1(1):85-89, 1990.

Received August 28, 2007 